\title{
Growth and Characterization of Pure and Doped L-Alanine Tartrate Single Crystals
}

\author{
K. Rajesh, B. Milton Boaz, and P. Praveen Kumar \\ Department of Physics, Presidency College, Chennai 5, India \\ Correspondence should be addressed to P. Praveen Kumar; ppkpresidency@gmail.com
}

Received 17 December 2012; Accepted 21 February 2013

Academic Editor: Alfonso Castiñeiras

Copyright (C) 2013 K. Rajesh et al. This is an open access article distributed under the Creative Commons Attribution License, which permits unrestricted use, distribution, and reproduction in any medium, provided the original work is properly cited.

\begin{abstract}
Single crystals of pure and Lanthanum doped L-Alanine Tartrate were grown by slow evaporation method. The cell parameters were determined using single crystal X-ray diffraction method. To improve the physical properties of the LAT crystal, Lanthanum dopant was added by $2 \mathrm{~mol} \%$. ICP studies confirm the presence of Lanthanum in the grown LAT crystal. Transparency range of the crystal was determined using UV-VIS-NIR spectrophotometer. The functional groups of pure and doped LAT crystals were analyzed by FT-IR spectroscopy. Using Vickers microhardness tester, mechanical strength of the material was found. Dielectric studies of pure and doped LAT single crystals were carried out. The doped LAT crystal is found to have efficiency higher than that of pure LAT crystal.
\end{abstract}

\section{Introduction}

In recent years organic nonlinear optical (NLO) crystals have attracted much attention for their large nonlinear coefficient, high laser damage threshold $[1,2]$. However, most of organic NLO materials have poor mechanical and thermal properties, resulting in the damage of crystal during processing. To avoid this drawback, a new type of NLO material has been grown from organic-inorganic complexes. These semiorganic materials have high optical nonlinearity of a purely organic compound combined with the mechanical and thermal properties of inorganic materials [3]. Amino acids have attracted a wide interest of the researchers, since all the compounds in the class consist of an optically active property. Thus, amino acids have special physical properties which make them an ideal candidate for their NLO applications [4]. Recently, LAlanine crystals have increased attention for photoinduced nonlinear optical effects and dispersion of the linear and nonlinear optical susceptibilities. Photoinduced nonlinear optical effects of L-Alanine single crystals show that the increasing time of illuminations leads to slight changes in the absorption backgrounds without changes in the spectral features [5]. Dispersion of the linear and nonlinear optical susceptibilities of L-Alanine single crystals was reported [6].
In the present work, a systematic study has been carried out on the growth of pure and Lanthanum doped LAlanine Tartrate (LAT) crystals. An attempt has been made to improve the physiochemical properties of LAT by adding Lanthanum dopant.

Single crystal X-ray diffraction study has been carried out to confirm the grown pure and doped crystals. ICP studies have been carried out to confirm the presence of Lanthanum in the grown crystal. FT-IR, UV-VIS-NIR, microhardness, dielectric analysis and NLO property were studied for grown pure and doped crystals. The second harmonic generation (SHG) studies had been carried out for the grown pure and doped crystals.

\section{Experimental Procedure}

Pure LAT crystal was grown by slow evaporation technique using water as a solvent. Analytical grade (AR) L-Alanine and Tartaric acid was taken in equimolar ratio. Saturated solution was prepared, filtered, and allowed to evaporate at room temperature under optimized conditions. Seed crystals were obtained in a period of one month. Good quality crystals were obtained by successively recrystallization method. The same procedure was applied to grow the metal doped crystals 


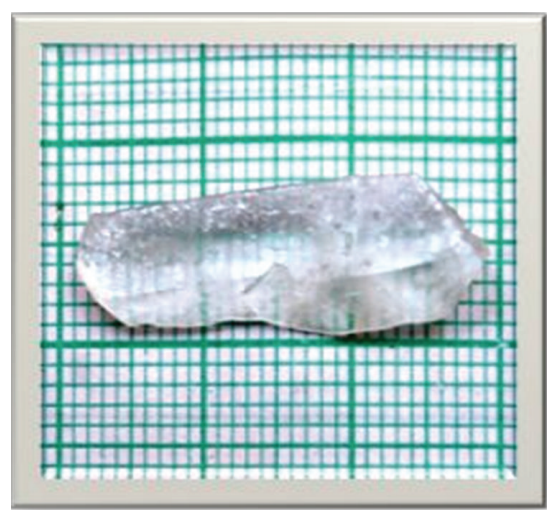

(a)

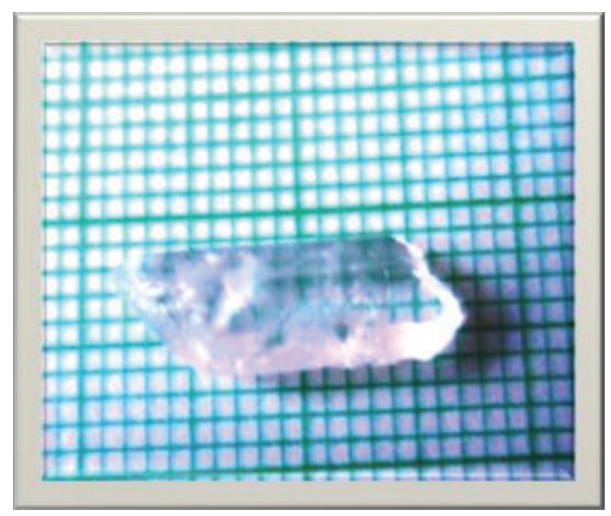

(b)

FIGURE 1: Photographs of pure and doped LAT crystals.

TABLE 1: Lattice parameter value for the pure and doped LAT crystals.

\begin{tabular}{lcc}
\hline Lattice parameters & Pure LAT & La $^{2+}$-LAT \\
\hline$a(\AA)$ & $5.790(\AA)$ & $5.823(\AA)$ \\
$b(\AA)$ & $6.386(\AA)$ & $6.372(\AA)$ \\
$c(\AA)$ & $12.157(\AA)$ & $12.121(\AA)$ \\
Crystal system & Monoclinic & Monoclinic \\
Space group & $\mathrm{P} 2_{1}$ & $\mathrm{P} 2_{1}$ \\
Volume $\left(\AA^{3}\right)$ & $449.504\left(\AA^{3}\right)$ & $449.70\left(\AA^{3}\right)$ \\
\hline
\end{tabular}

by adding $2 \mathrm{~mol} \%$ of Lanthanum to the LAT solution. Good quality crystals were obtained in a period of three weeks. The incorporation of dopant into the pure solution has improved the growth rate and the quality of the crystals. The grown crystal was further studied by various characterization techniques. The photographs of grown pure and Lanthanum doped crystals are shown in Figure 1.

\section{Results and Discussion}

3.1. Single Crystal X-Ray Diffraction Studies. Single crystal Xray diffraction studies of pure and Lanthanum doped LAT crystals were carried out using MESSRS ENRAF NONIUS CAD4-F, single X-ray diffractometer. Both the pure and doped LAT crystals belong to monoclinic crystal system with the space group $\mathrm{P} 2{ }_{1}$. The lattice parameter values of pure and doped crystals were also tabulated.

There is a slight variation in the lattice parameters of doped LAT compared with pure LAT. These variations are due to the incorporation of Lanthanum in the LAT crystal lattice. The lattice parameters of the pure and doped crystals are shown in Table 1.

3.2. ICP Studies. The exact weight percentage of the $\mathrm{La}^{2+}$ present in the doped crystal is determined.

$10 \mathrm{mg}$ of fine powder of the doped LAT crystal was dissolved in $30 \mathrm{~mL}$ of doubled distilled water, and the prepared sample was subjected to ICP analysis. The result shows that only $0.5 \%$ of $\mathrm{La}^{2+}$ is present in the sample out of $2 \%$ of the dopant. It is seen that the amount of dopant incorporated in to the doped crystal is less than the concentration of the dopant in the corresponding solution.

3.3. Fourier Transform-Infrared Spectroscopy (FT-IR). The fine powdered samples of pure and doped LAT crystals are taken for this experiment. The FT-IR spectrum is recorded for the pure and doped LAT single crystals at room temperature in the range of $400-4000 \mathrm{~cm}^{-1}$ by employing BRUKKER IFS $66 \mathrm{~V}$ FT-IR spectrometer, using KBr pellet method. The FT-IR spectra of pure and doped crystals are shown in Figure 2.

The $\mathrm{OH}$ stretching vibrations were observed for both of the pure and metal doped LAT crystals at $3085 \mathrm{~cm}^{-1}$. Both of the pure and doped metal doped compounds show strong absorption at $2601 \mathrm{~cm}^{-1}$ indicating the presence of amine group. The absorption at $2111 \mathrm{~cm}^{-1}$ is due to $\mathrm{NH}_{3+}$ asymmetric deformation and $\mathrm{NH}_{3+}$ hindered rotation. It shows that addition of metal dopant do not show any significant from the pure crystal spectrum.

3.4. UV Analysis. The UV absorption study of the pure and doped LAT crystals was carried out by UV-VIS-NIR spectrophotometer. Figure 3 shows the maximum absorption of pure and doped LAT crystals starts from $203 \mathrm{~nm}$. After this wavelength, absorption abruptly decreases to nearly $2 \%$ for pure and $1.5 \%$ for doped LAT crystals. The lower cut-off wavelength of pure and doped crystals occurs at $246 \mathrm{~nm}$ and $235 \mathrm{~nm}$, respectively. Thus it is clear that both of materials possess very good optical transparency up to $1500 \mathrm{~nm}$. The doped crystal has better transparency window compared to the pure one. This property makes the material suitable for the many potential and nonlinear applications.

3.5. Microhardness Study. Microhardness studies of pure and doped LAT crystals have been carried out using HMV SHIMADZU microhardness tester, fitted with diamond Vickers pyramidal indenter. The microhardness measurements are carried out on the well-developed face $\left(\begin{array}{lll}1 & 0 & 0\end{array}\right)$. The static indentations were made at room temperature with a constant 


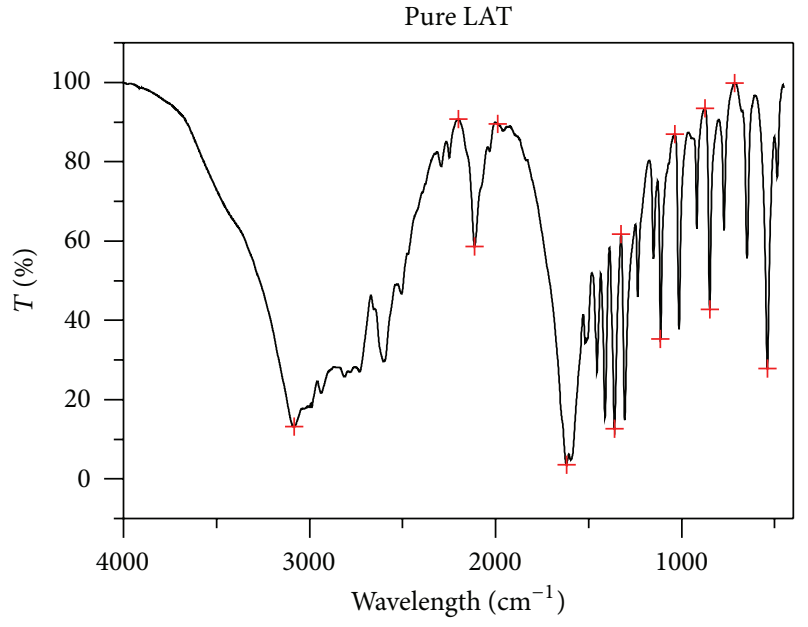

(a)

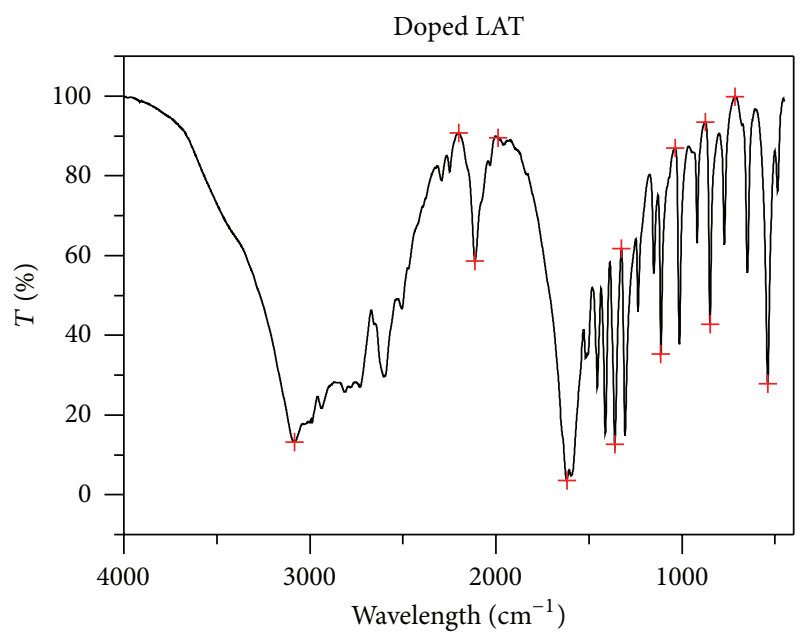

(b)

FIGURE 2: The FT-IR spectra of pure and doped crystals.

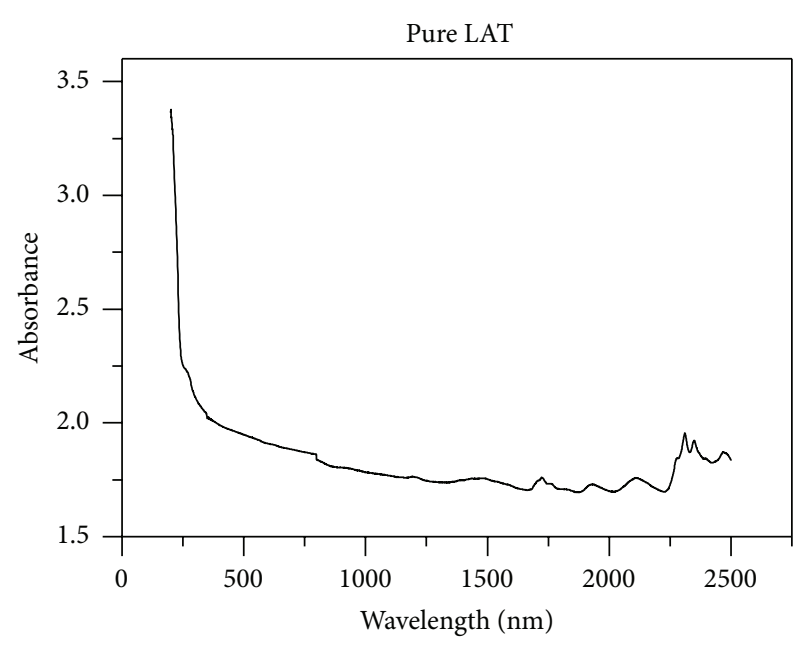

(a)

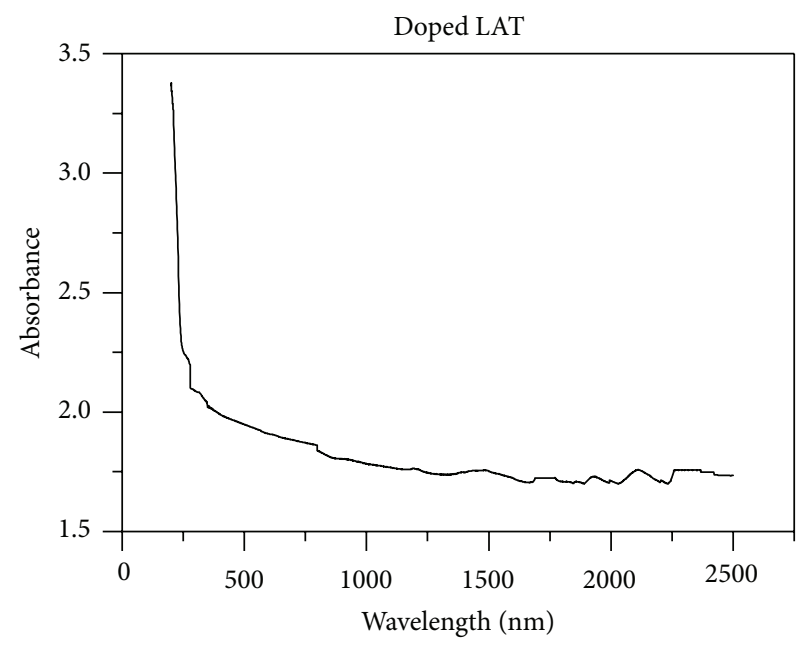

(b)

FigURE 3: UV-VIS-NIR spectra of pure and doped LAT crystals.

indentation time of 15 seconds for all indentations. Maximum indenter load applied for LAT crystal was $50 \mathrm{~g}$, and above this load microcracks are observed. The plot of variation of Vickers hardness number with applied load for the plane $\left(\begin{array}{lll}1 & 0 & 0\end{array}\right)$ of pure and doped LAT crystals is shown in Figure 4.

For pure LAT crystal the hardness value increases as load increase up to $45 \mathrm{~g}$. Beyond this load cracks were found to develop. Thus the pure material is mechanically stable up to the load of $45 \mathrm{~g}$. In metal doped LAT crystal the cracks were found when the load value is $50 \mathrm{~g}$. This indicates that the metal dopant in the compound increases the mechanical hardness of the material. By plotting $\log p$ verses $\log d$, the value of the working hardness coefficient $n$ value for pure and doped LAT crystals was found to be 1.38 and 1.27. According to Onitsch, $n$ lies between 1 and 1.6 for hard materials and $n$ is greater than 1.6 for soft materials [7]. Hence, it is concluded that the pure and doped LAT crystals belong to hard material category.

3.6. Dielectric Analysis. The dielectric study of pure and doped LAT single crystals was carried out using the HIOKI 3532-50 LCR HITESTER instrument. The capacitance values for both pure and doped crystals are found for frequencies varying from $50 \mathrm{~Hz}$ to $5 \mathrm{MHz}$ at room temperature.

Figure 5 shows the variations of dielectric constant with $\log$ frequency for pure and doped LAT crystals. It is observed from the plot that the dielectric constant decreases with increasing frequency and attains a constant value in the high frequency region. At room temperature, the dielectric constant decreases with applied frequency. From the graph it is clearly evident that the $\mathrm{La}^{2+}$ doped LAT crystal shows 


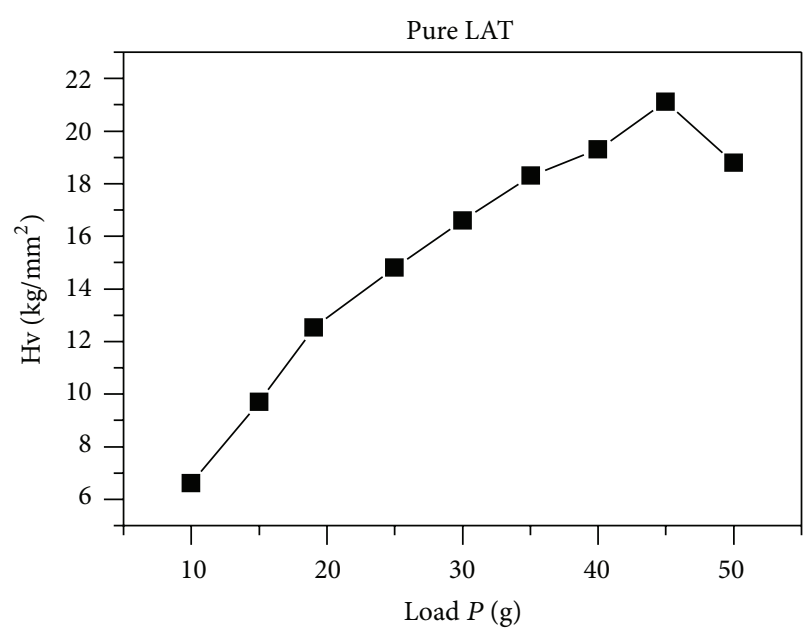

(a)

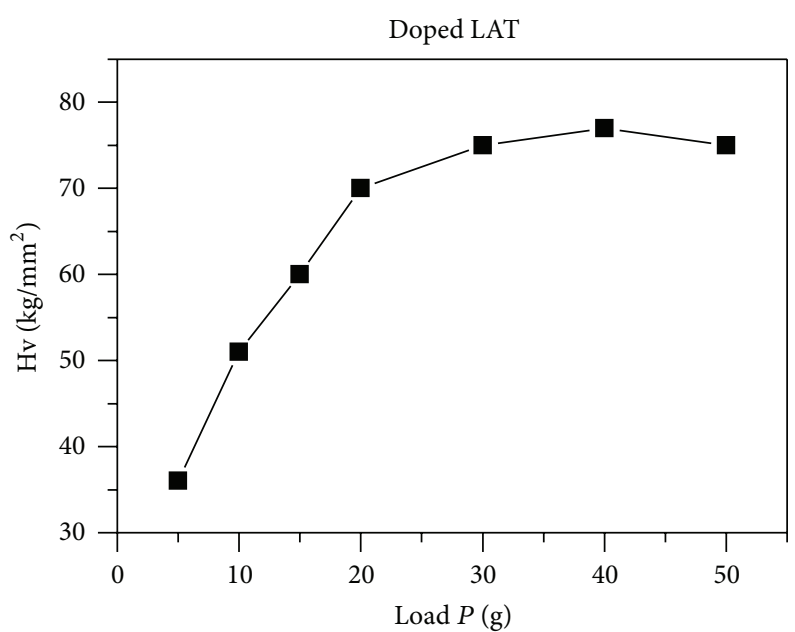

(b)

FIGURE 4: The plot of variation of Vickers hardness number with applied load for the plane $\left(\begin{array}{lll}1 & 0 & 0\end{array}\right)$ of pure and doped LAT crystals .

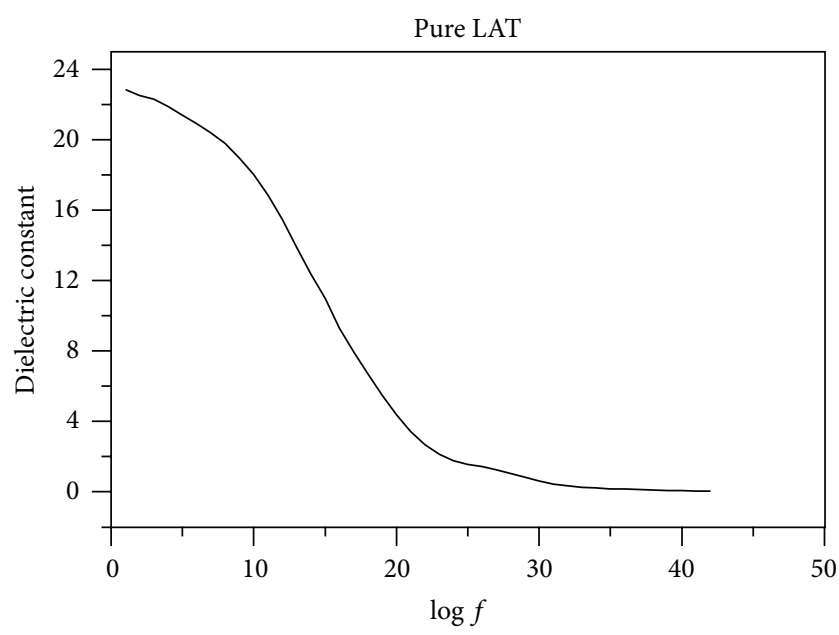

(a)

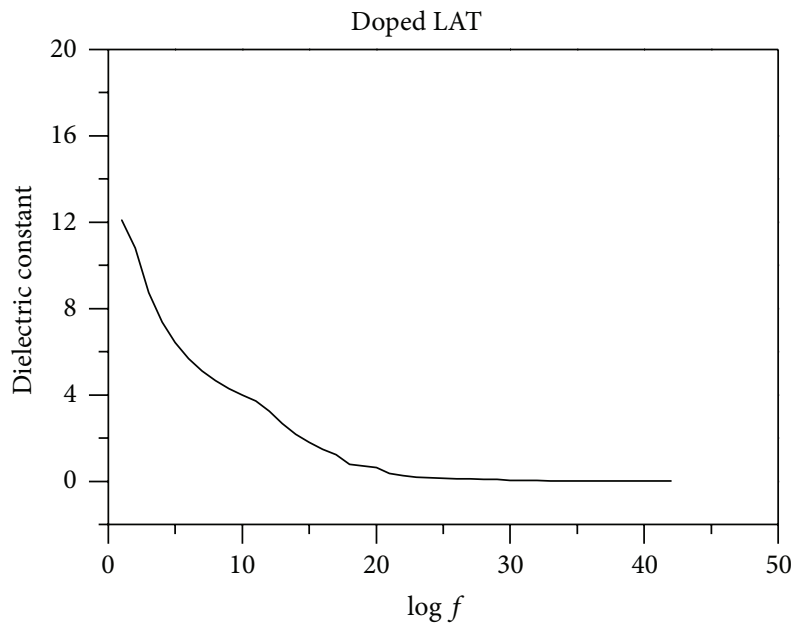

(b)

FIGURE 5: Dielectric constant with log frequency for pure and doped LAT crystals.

more efficiency than the pure one. Thus the dielectric nature of pure LAT is altered by the presence of $\mathrm{La}^{2+}$ metal.

In $\mathrm{La}^{2+}$ doped LAT crystal the low dielectric constant with high frequency suggests that the crystal possesses enhanced optical quality with lesser defects and this parameter is of vital importance for various nonlinear optical materials and their applications [8].

3.7. NLO Studies. Nonlinear optical (NLO) property of pure and doped LAT crystals was carried out from Kurtz powder second harmonic generation (SHG) test using the Nd: YAG Q-switched laser beam. The sample of same size was illuminated using Q-switched, mode locked Nd:YAG laser with input pulse of $6.2 \mathrm{~mJ}$.

The second harmonic signals of $324 \mathrm{mV}$ and $384 \mathrm{mV}$, respectively, were obtained for pure and doped LAT crystals with reference to KDP $(275 \mathrm{mV})$. Thus, the SHG efficiency of pure and doped LAT crystals is nearly 1.18 and 1.40 times greater than KDP. The doped LAT crystal is found to have efficiency higher than that of pure LAT. The increase in the SHG efficiency of the doped crystals is due to the presence of metal dopant in the crystal lattice [9]. It is concluded that the doped LAT crystal can be used for desired NLO applications.

\section{Conclusion}

Single crystals of pure and Lanthanum doped L-Alanine Tartrate (LAT) were grown successfully by slow evaporation technique. Single crystal X-ray diffraction studies were carried out, and the lattice parameters are tabulated. ICP studies confirm the presence of the dopant in the grown crystal. UV-VIS-NIR studies reveal that the pure and doped crystals 
possess wide transparency in the entire visible region. FTIR studies reveal the presence of various functional in the crystal, and vibrational structure of the compound has been elucidated. Hardness of both pure and doped LAT crystals reveals that these crystals are hard materials. The dielectric studies reveal that the dielectric constant decreases with log frequency. NLO studies proved that the Lanthanum dopant has increased the efficiency of pure LAT. Thus it is concluded that the presence of dopant has improved the NLO properties of the grown crystals.

\section{References}

[1] R. Ittyachan and P. Sagayaraj, "Growth and characterization of a new nonlinear optical l-histidine diphosphate single crystal," Journal of Crystal Growth, vol. 249, pp. 553-556, 2003.

[2] R. W. Boyd, Nonlinear Optics, Academic Press, San Diego, Calif, USA, 1992.

[3] T. Pal and T. Kar, "Single crystal growth and characterization of the nonlinear optical crystal L-arginine hydrofluoride," Journal of Crystal Growth, vol. 234, no. 1, pp. 267-271, 2002.

[4] M. Narayan Bhat and S. M. Dharmaprakash, "Growth of nonlinear optical $\gamma$-glycine crystals," Journal of Crystal Growth, vol. 236, no. 1, pp. 376-380, 2002.

[5] A. Wojciechowski, K. Ozga, A. H. Reshak et al., "Photoinduced effects in l-alanine crystals," Materials Letters, vol. 64, no. 18, pp. 1957-1959, 2010.

[6] A. H. Reshak, S. Auluck, D. Stys et al., "Experimental and theoretical investigations," Journal of Materials Chemistry, vol. 21, p. 17219, 2011.

[7] S. Mukerji and T. Kar, "Vicker's microhardness studies of Larginine hydrobromide monohydrate crystals (LAHBr)," Crystal Research and Technology, vol. 34, no. 10, pp. 1323-1328, 1999.

[8] C. Balarew and R. Duhlew, "Application of the hard and soft acids and bases concept to explain ligand coordination in double salt structures," Journal of Solid State Chemistry, vol. 55, no. 1, pp. 1-6, 1984.

[9] P. Praveen Kumar, V. Manivannan, S. Tamilselvan et al., "Growth and characterization of a pure and doped nonlinear optical L-histidine acetate single crystals," Optics Communications, vol. 281, no. 10, pp. 2989-2995, 2008. 

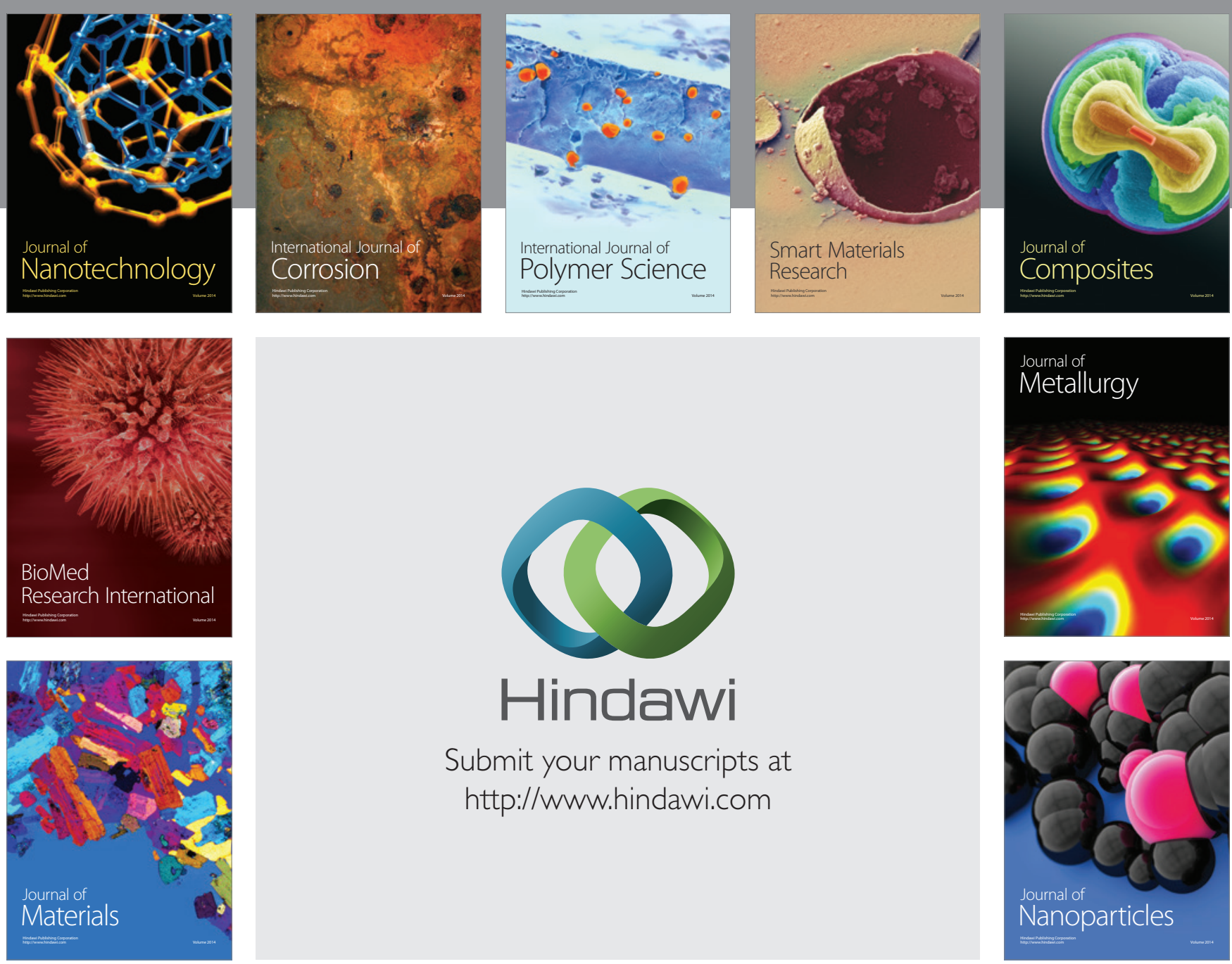

Submit your manuscripts at http://www.hindawi.com
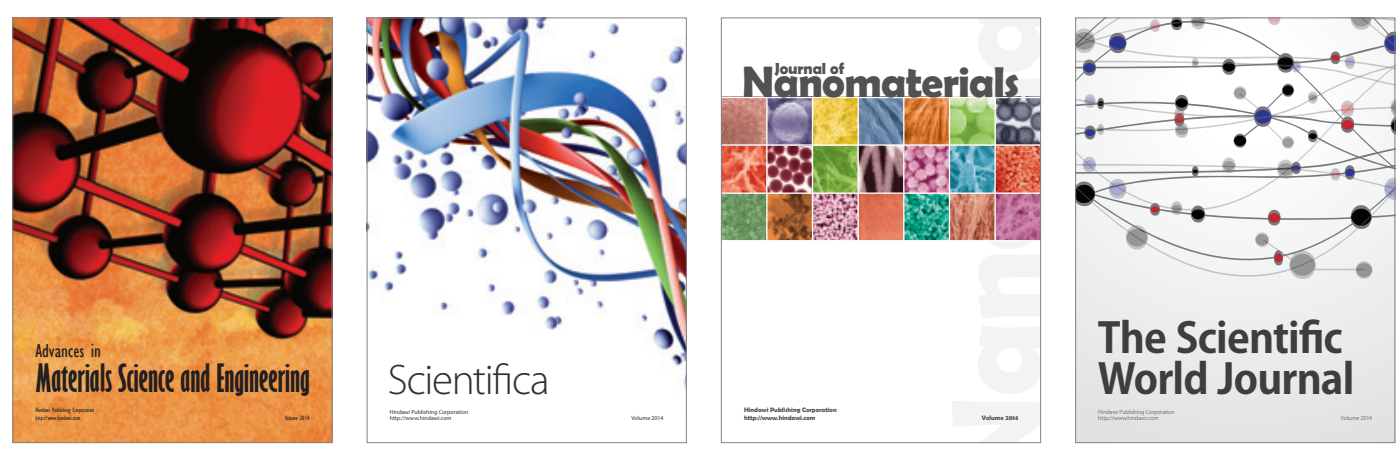

\section{The Scientific World Journal}
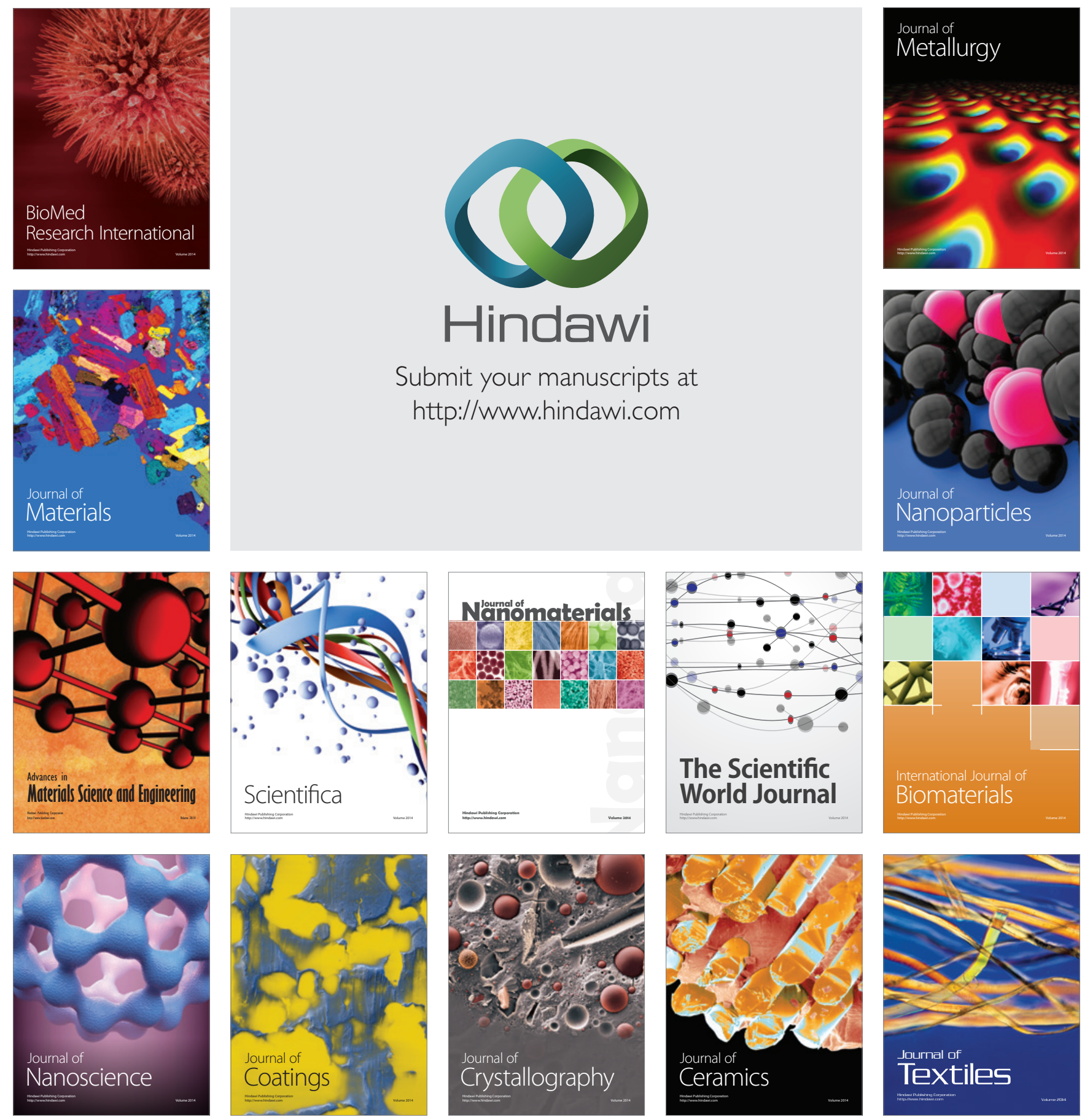